\title{
Double-Minute Chromosomes in the Leukocytes of a Patient with a Previous History of Cervical Carcinoma
}

\author{
A. B. Mitra, V. V. V. S. Murty, and Usha K. Luthra
}

ABSTRACT: Double-minute chromosomes (DMs) were observed in repeated samples in the leukocytes of a patient with a previous history of cervical carcinoma. The most interesting cytogenetic finding was the coexistence of DMs and a dicentric chromosome along with chromosome-and chromatid-type breaks and gaps. This observation suggests that DMs might originate through the breakage of existing chromosomes. The presence of DMs in leukocytes may also indicate the possibility that certain common agents cause DMs in tumor cells as well as in normal cells.

\section{INTRODUCTION}

Double minutes (DMs) are considered a unique phenomenon in tumor biology, although their origin and significance are not yet clearly known. Several reports have been published on the presence of DMs in various tumors [1-5], particularly in neurogenic tumors [6-12]. Recently, DMs were reported in cervical carcinoma cells [2]. They have been reported both in vivo and in vitro in breast tumor cells [1]. So far, there is only a single case report wherein DMs were observed in peripheral leukocytes of a patient with neuroblastoma [6]. The presence of DMs in cells other than tumor cells points to a possible common agent(s) responsible for producing DMs in tumor cells and other cells of the body. More data are needed to generalize this view. This article reports the presence of DMs in peripheral leukocytes in a patient having a previous history of treated cervical cancer.

\section{MATERIAL AND METHODS}

\section{Case Report}

The patient, aged 48 years, had a history of cervical carcinoma of the uterine cervix. Her disease had been clinically diagnosed as stage III, and she had undergone radiotherapy (6000R) which included two insertions of radium spaced at an interval of 1 week and followed by 15 external x-ray exposures in the parametrial region 5 years before. She reported to the hospital for a routine checkup, and peripheral blood was collected for the first time for cytogenetic analysis. The sample was repeated after 1 year. At present, the patient has no clinical evidence of carcinoma. Cytological examination of the cervical smear did not reveal any evidence of malignancy.

From the Cytology Research Centre (ICMR), Maulana Azad Medical College, New Delhi, India.

Address requests for reprints to Dr. A. B. Mitra, Cytology Research Centre, Maulana Azad Medical College, Bahadur Shah Zaffar Marg, New Delhi-110 002, India.

Received February 25, 1982; accepted June 16, 1982. 
Peripheral blood leukocytes were cultured according to a conventional method in the presence of phytohemaglutinin (Gibco) for $72 \mathrm{hr}$. Leukocytes were also cultured in the presence of bromodeoxyuridine (BrdU) for sister chromatid exchange (SCE) analysis. In the first and second samples, respectively, 65 and 90 metaphase plates were examined for the study of chromosomal aberrations.

\section{RESULTS}

The most important cytogenetic observation was the presence of DMs and dicentric chromosomes and their existence in a subsequent repeat sample after 1 year. The cytogenetic analysis revealed a similarity in the types of chromosomal aberrations observed in both samples. However, they varied quantitatively (Table 1).

In the first sample, out of 65 metaphase plates, $4(6.2 \%)$ revealed DMs. Of the 4 metaphase plates containing DMs, 2 had a dicentric chromosome (Fig. 1). In both plates the chromosome count was 45 . The number of DMs varied between one and three. Variation in the size of the DMs was also observed. In conventional Giemsa staining the DMs were stained similarly to other chromosomes. In BrdU-treated cultures, they showed differential staining of sister chromatids (Fig. 2). In addition to DMs and dicentric chromosomes, other chromosomal aberrations (chromatid breaks, gaps, and acentric fragments) were observed (Fig. 3). The frequency of different chromosomal aberrations varied (Table 1). The most frequent aberration was chromatid-type breaks $(9.2 \%)$, which were of random nature. The average frequency of SCEs per metaphase was 7.8, and no heteromorphism of C-bands was observed.

In the second sample, out of 90 metaphase plates analyzed, 3 revealed DMs in varied numbers, and 1 of these showed a dicentric chromosome with DMs. Other chromosomal aberrations observed in the sample are listed in Table 1. It is interesting to note the similar nature and frequency of the chromosomal aberrations, including DMs and dicentric chromosomes, even after 1 year.

\section{DISCUSSION}

An interesting feature of the cytogenetic observations in this case was the presence of DMs and dicentric chromosomes in the leukocytes of peripheral blood and their persistence. The presence of DMs in peripheral leukocytes has been reported only in a single case of neuroblastoma [6]. There is no report on the presence of DMs in peripheral leukocytes of normal individuals. The present evidence supports the possibility of a common agent(s) that may cause the formation of DMs in tumor cells as well as in peripheral leukocytes.

There are several different explanations regarding the origin of DMs: (1) DMs are fragments of preexisting chromosome regions; (b) DMs are a result of the amplifi-

Table 1 Frequency of chromosomal aberrations in leukocytes of a patient with a previous history of cervical carcinoma

\begin{tabular}{cccccc}
\hline \multirow{2}{*}{$\begin{array}{l}\text { Date of } \\
\text { examination }\end{array}$} & $\begin{array}{l}\text { No. of } \\
\text { metaphases }\end{array}$ & Chromosome & Chromatid & & \multicolumn{2}{c}{$\begin{array}{l}\text { Metaphases } \\
\text { with } \\
\text { abnormalities }\end{array}$} \\
\cline { 5 - 6 } $5 / 6 / 81$ & 65 & 3 & 10 & 4 & 10 \\
$22 / 5 / 82$ & 90 & $(4.6)$ & $(15.4)$ & $(6.2)$ & $(15.4)$ \\
& & 4 & 12 & 3 & 11 \\
& & $(4.4)$ & $(13.3)$ & $(3.3)$ & $(12.2)$ \\
\hline
\end{tabular}

Figures in parentheses are percentages. 


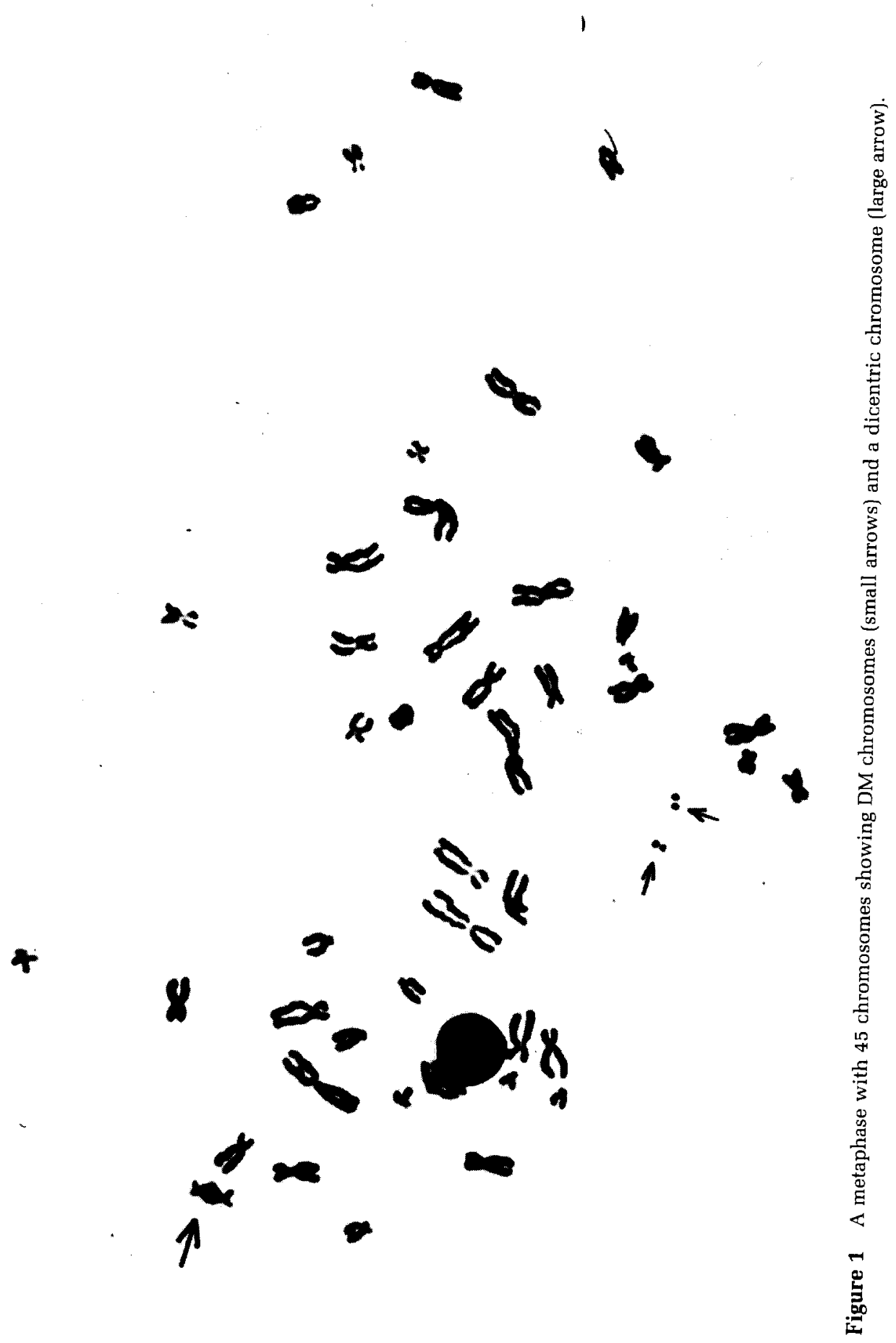




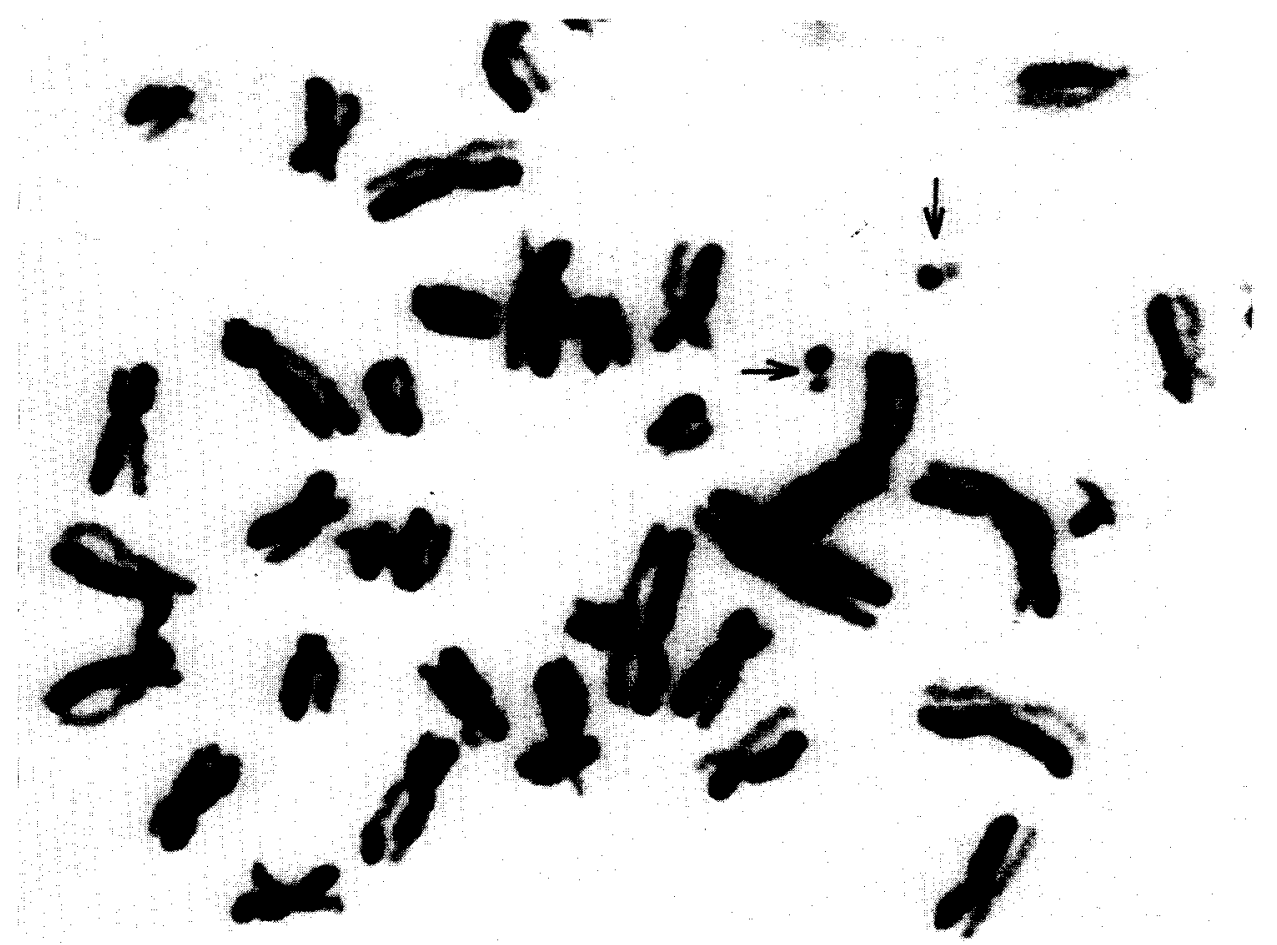

Figure 2 A partial metaphase showing differential staining of sister chromatids of DM chromosomes.

cation of subchromosomal regions; and (c) DMs are extrachromosomal in nature [1]. Based on our material, it is evident that DMs originate from existing chromosomes and are not extrachromosomal bodies, as both conventional Giemsa staining and BrdU treatment with staining for sister chromatids revealed DMs to be identical to those from other chromosomes.

Another interesting observation was the presence of dicentric chromosomes along with DMs. So far, no report is available on the presence of both DMs and dicentric chromosomes in the same metaphase. This observation strongly supports the possibility that the dicentric chromosomes and DMs in the present case originated through the breakage and fusion of conventional chromosome regions. Two pairs of DMs and one dicentric chromosome in one metaphase plate suggest this as the most possible explanation. Leukocytes grown in vitro for a long time indicated the possibility that the formation of DM-like structures in several areas of different chromosomes was a degenerative effect [6].

The number of DMs per cell did not show any correlation with the number of chromosomes or any other abnormality. DMs in cells with a normal diploid chromosome number have been reported by several other authors $[1,6]$. Elimination of acentric fragments generally occurs in subsequent cell divisions, and DMs would be expected to be eliminated if they were chromosome fragments. However, based on the available literature and on present evidence, the variation in the distribution of DMs in various cells reflects their random distribution but does not support their gradual elimination because of the absence of a centromere. DMs frequently form clusters outside the equatorial plate during anaphase, and these DM clusters move passively with the chromosomes $[1,6,10,13,14]$. It has also been postulated that the 


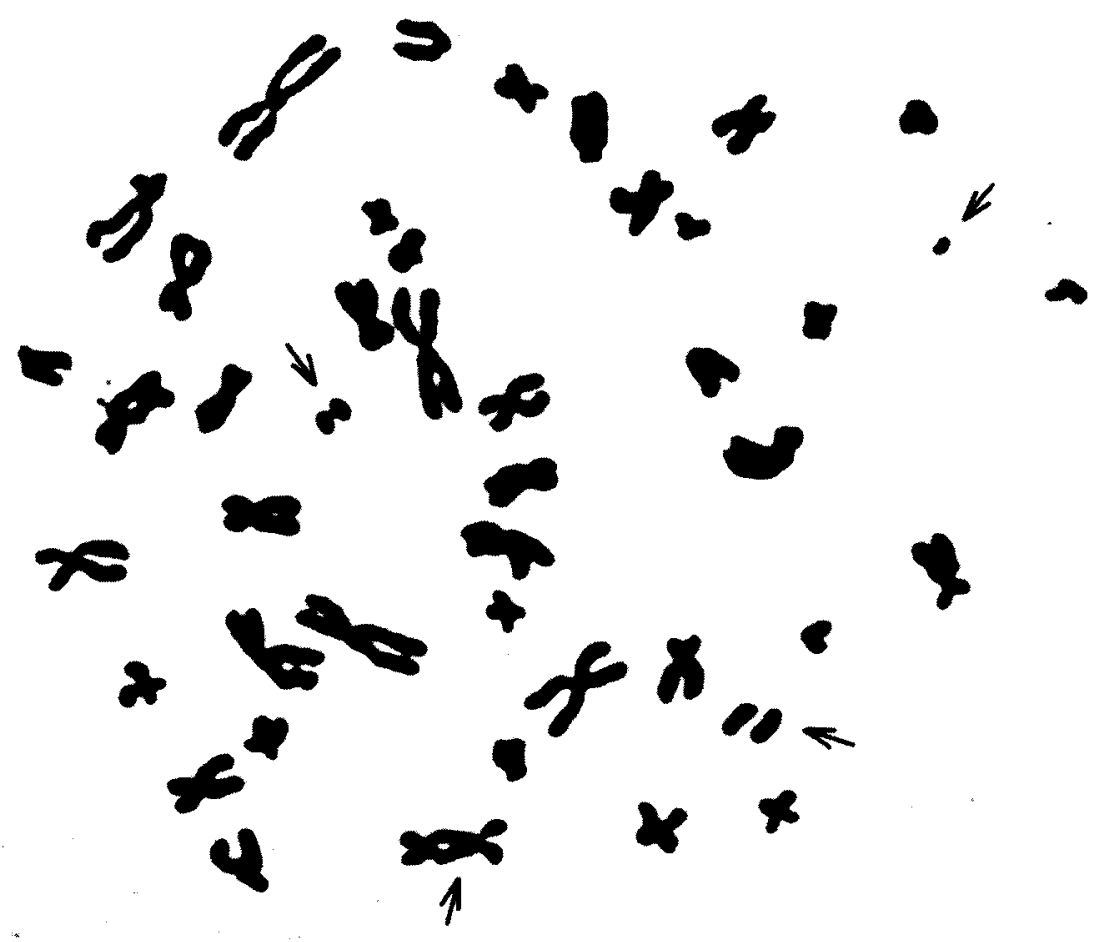

Figure 3 A partial metaphase with various aberrations: a dicentric chromosome, DMs, acentric fragments.

maintenance of DMs in a cell population without gradual elimination is possible through the formation of micronuclei which then undergo mitosis synchronously. However, most possibly, variation in the number of DMs occurs mainly through nondisjunction and/or replication phenomenon. The possible time of origin of DMs is controversial. The observation of dicentric chromosomes and chromatid-type breaks in our material suggests the possibility that DMs originate during or after the replication phase and not in the $\mathrm{G}_{2}$-phase, as previously reported [6].

The patient had a history of radiotherapy. It is well established that radiation causes various chromosomal aberrations. However, it is difficult to attribute the formation of DMs only to radiation effects, since DMs and dicentric chromosomes could not be observed in any patients with a history of radiotherapy studied in the laboratory.

Spontaneous regression of neuroblastoma and other neurogenic tumors have been reported to be common, although the actual mechanism is not known. Possible association of DMs with the regression of tumors has been suggested by Sandberg et al. [6] in view of the fact that DMs are commonly seen in these tumors. Clinical examination of the case revealed complete regression of cancer after radiotherapy 5 years before. At present it is very difficult to make any comment on the significance of DMs in the peripheral lymphocytes of a patient with cervical cancer from a diagnostic and prognostic point of view. However, more data are needed for further comment in this regard.

The authors are grateful to Mr. K. L. Katyal for secretarial help and to Mrs. Raj Menon and Mrs. S. Kaur for collecting material. 


\section{REFERENCES}

1. Barker PE, Hsu TC (1979): Double minutes in human carcinoma cell lines, with special reference to breast tumours. Cancer $62,257-261$.

2. Atkin NB, Baker MC (1980): Cytogenetic observations on a carcinoma of the cervix uteri with double minute chromatin bodies. Eur J Cancer 16, 793-797.

3. Pierre RV, Hoagland KC, Linman JW (1971): Microchromosomes in human preleukemia and leukemia. Cancer 27, 160-175.

4. Atkin NB, Pickthall VJ (1977): Chromosome 1 in 14 ovarian cancers: Heterochromatin variants and structural changes. Hum Genet 38, 25-33.

5. Miles CP (1967): Chromosome analysis of solid tumours. II. Twenty-six epithelial tumours. Cancer 20, 1274-1279.

6. Sandberg AA, Sakurai M, Holdsworth RN (1972): Chromosomes and causation of human cancer and leukemia. VIII. DM chromosomes in a neuroblastoma. Cancer 29, 1671-1679.

7. Cox D, Yuncken C, Spriggs AI (1965): Minute chromatin bodies in malignant tumours of childhood. Lancet ii, 55-58.

8. Levan A, Manolov G, Clifford P (1968): Chromosomes of a human neuroblastoma : A new case with accessory minute chromosomes. J Natl Can Inst 41, 1377-1387.

9. Hansteen IL (1967): Chromosome studies in glial tumors. Eur J Cancer 3, 183-191.

10. Mark J, Granberg I (1970): The chromosomal aberrations of double minutes in three gliomas. Acta Neuropathol 16, 194-204.

11. Biedler JL, Helson L, Spengler BA (1973): Morphology and growth, tumorigenicity and cytogenetics of human neuroblastoma cells in continuous culture. Cancer Res 33, 26132652.

12. Balaban-Malenbaum G, Gilbert F (1977): Double minute chromosomes and homogeneously staining regions in chromosomes of a human neuroblastoma cell line. Science $198,739-742$.

13. Mark, J (1971): Chromosomal characteristics of neurogenic tumours in adults. Hereditas 68, 61-100.

14. Levan A, Levan $G$ (1978): Have double minutes functioning centromeres? Hereditas 88, 81-92. 